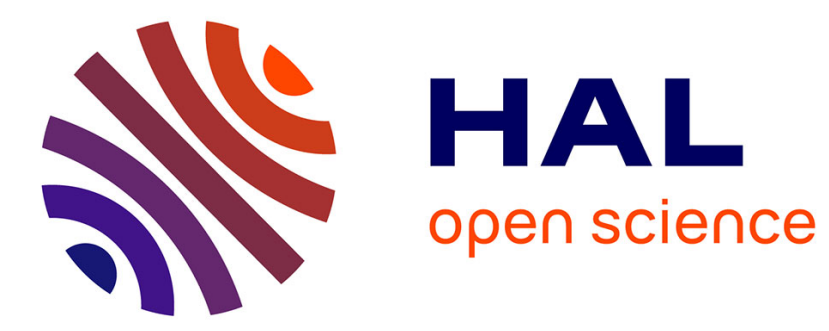

\title{
Haptic feedback for molecular simulation
}

Aude Bolopion, Barthelemy Cagneau, Stephane Redon, Stéphane Régnier

\section{To cite this version:}

Aude Bolopion, Barthelemy Cagneau, Stephane Redon, Stéphane Régnier. Haptic feedback for molecular simulation. IROS 2009 - IEEE/RSJ International Conference on Intelligent Robots and Systems, Oct 2009, St. Louis, MO, United States. pp.237-242, 10.1109/IROS.2009.5354256 • hal-00784678

\section{HAL Id: hal-00784678 \\ https://hal.inria.fr/hal-00784678}

Submitted on 14 Feb 2013

HAL is a multi-disciplinary open access archive for the deposit and dissemination of scientific research documents, whether they are published or not. The documents may come from teaching and research institutions in France or abroad, or from public or private research centers.
L'archive ouverte pluridisciplinaire HAL, est destinée au dépôt et à la diffusion de documents scientifiques de niveau recherche, publiés ou non, émanant des établissements d'enseignement et de recherche français ou étrangers, des laboratoires publics ou privés. 


\title{
Haptic feedback for molecular simulation
}

\author{
Aude Bolopion, Barthélemy Cagneau, Stéphane Redon and Stéphane Régnier
}

\begin{abstract}
In this paper, a new tool dedicated to the analysis and the conception of molecules is presented. It is composed of an adaptive simulation software and a haptic device used to interact with molecules while feeling either the forces applied by the environment or the internal forces. The adaptive articulated body algorithm allows fast simulations of complex flexible molecules. To handle the coupling with the force feedback device, two different control schemes designed for nanoscale applications and providing high transparency rendering are proposed and compared.

The system we propose is highly flexible since either a single rigid body or the entire molecule can be manipulated via the haptic device. The user can choose between setting a desired position/orientation of the molecule, or apply forces/torques to manipulate it. It allows the operator to control each stage of the design process of new molecular structures.

The validity of this tool is demonstrated through examples of haptic interaction between the HIV protease and its inhibitors, and unfolding one of these drugs.
\end{abstract}

\section{INTRODUCTION}

Molecular mechanics simulators are efficient tools to study molecular interactions and docking, with applications in many fields, including pharmacology, cosmetics, nanosciences and surface physics. In particular, interactive molecular simulations may be a significant help to understand molecular interactions. For these simulations, a user can control the motion of one or more atoms to guide the evolution of the molecular system.

Most of the work deal with molecular docking. In [1], the authors explain how the user's intervention may facilitate the docking, by controlling the position of the ligand and feeling the interaction forces. Other studies have then been carried out. The simulator and the bilateral couplings proposed differ in many ways. For example, in the simulation, the force field can be computed thanks to the derivation of the potential energy [2]. Even if some simulators take into account the flexibility of the ligand and the receptor [3], most of them do not [4]. Concerning the coupling, wave variables are also used to guarantee the passivity of the system even when time delays increase [5]. However, this method may lead to a lack of transparency.

Besides molecular docking, other emerging fields of research concern molecular interactions. The molecules can be used as a channel to transmit an information between two nanomachines ([6] and [7]). Another research field is the

A. Bolopion, B. Cagneau, S. Régnier are with Institut des Systèmes Intelligents et de Robotique, Université Pierre et Marie Curie, CNRS UMR 7222, 4 Place Jussieu, 75005 Paris, France. \{bolopion, cagneau, regnier\}eisir.fr

S. Redon is with NANO-D - INRIA Rhône-Alpes, 655 avenue de l'Europe, 38334 Saint-Ismier Cedex, France stephane.redon@inria.fr study of molecules in order to develop biomicroelectromechanical system devices (bio-MEMS) ([8] and [9]).

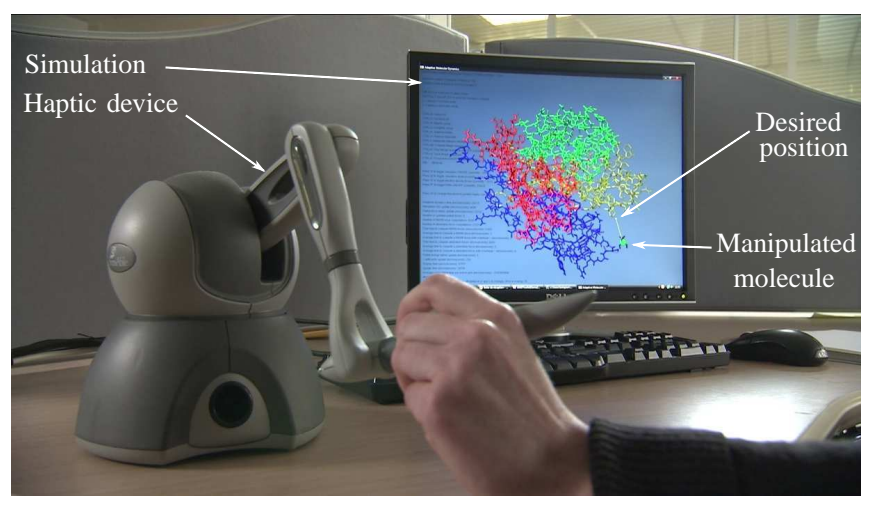

Fig. 1. Manipulation of a tetraethylamonium (TEA) around a potassium channel (KcsA). A haptic device is used to control the molecule and feel the interactions between the ligand and the protein derived thanks to the adaptive simulation

Tools to deal with specific applications have been developed. However, emerging applications as the conception of new materials and alloys, or molecules with specific properties require more flexibility. Both the internal structure of the molecule and the interactions with its environment must be understood. The manipulation of the molecule must be done in real time, and be intuitive for users with no training. Thus, we propose a system, composed of a molecular simulation software and a haptic interface, able to deal with complex flexible molecules in real time (figure 1). The simulation software is based on adaptive simulation of articulated body, so that the computation time is decreased dramatically. The algorithm used has already been exposed in [10] and [11] Both internal forces and interactions between the molecule and its environment are computed. To interact with the simulation, a Phantom Omni haptic device is used to manipulate the molecule, and render forces to the user. To handle the coupling between the haptic device and the simulation, two different control schemes are implemented. The user can manipulate a single rigid body, or an entire molecule. $\mathrm{He}$ or she can either set the desired position/orientation, or apply force/torque to control the object. We performed several experiments using this system. Interactions between the HIV protease and its inhibitors are used to compare the two control schemes. The feeling of internal forces is demonstrated by unfolding one of its inhibitors.

This paper is organized as follows. In section II, the molecular simulator is briefly introduced. The two control schemes designed for nanoscale applications are presented 
in section III. In section IV, examples of manipulations that can be handled by our tool are presented. The advantages and drawbacks of each control scheme are also discussed. Finally, concluding remarks are exposed in section V.

\section{MOLECULAR MECHANICS}

In this paper, we use an adaptive molecular quasi-statics method to simulate the motion of the molecular system [11]. The adaptive method relies on a recursive, divide-andconquer representation of the molecular system, where any non-rigid group of atoms is considered to be the union of two groups of atoms. Thus, any molecular system is associated to a binary assembly tree, in which the leaf nodes represent the user-defined rigid bodies, the internal nodes represent the sub-assemblies, and the root node represents the complete molecular system (figure 2).

$$
\text { a }
$$

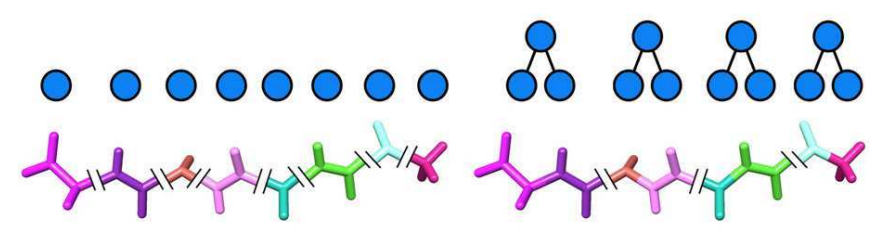

C
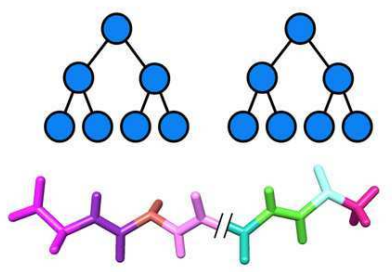

d

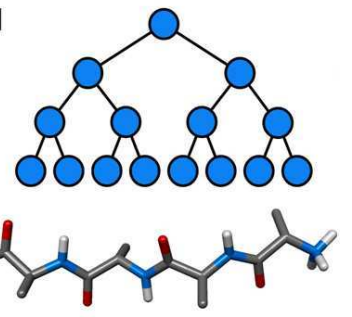

Fig. 2. The binary assembly tree of a tetra-alanine.

The distinctive feature of the adaptive algorithm is that it allows a user to finely trade between precision and computational cost. Precisely, the user may arbitrarily choose the number of degrees of freedom that are simulated at each time step, and the adaptive method automatically determines which degrees of freedom are activated at each time step (a sub-tree of the binary assembly tree), so as to approximate as faithfully as possible the motion which would have been obtained by a non-adaptive simulator. In practice, the adaptive algorithm focuses the computational resources on the joints with the largest accelerations, that is, the most flexible regions. Using this approach, complex molecular systems may be simulated on cheap personal computers while retaining molecular flexibility. In docking applications, the adaptive simulator automatically focuses the motions computations on the docking site.

Besides adaptivity, the simulator follows traditional approaches: at each time step, the interatomic forces are first computed, then the acceleration of the system is determined and used to update its state.
A user may influence the state of the molecular system by either directly controlling the position and orientation of a selected molecule, or by applying a force and torque to a group of atoms. In the latter case, the selected group of atoms may be either a complete molecule (e.g. an inhibitor of an HIV protease model), or a rigid body from a molecule.

The force fed back to the user is the total force applied by the molecular system to the group of atoms selected by the user. These forces directly depend on the force field being used in the simulation to model interatomic interactions. In our current implementation, the force field is derived from a well known molecular mechanics force field, CHARMM [11], which models interactions through van der Waals, electrostatic and dihedral contributions.

- Molecule. Assume the user manipulates an entire molecule $\mathcal{M}$, composed of rigid bodies $\mathcal{R}_{1}, \ldots, \mathcal{R}_{n}$. In this case, we determine the molecular forces $\mathbf{F}_{1}, \ldots \mathbf{F}_{n}$ applied on each rigid body by the other rigid bodies in the environment (i.e. van der Waals and electrostatic contributions from surrounding atoms, including those resulting from other rigid bodies in the selected molecule), and return to the user the $\operatorname{sum} \mathbf{F}=\sum_{i=1}^{n} \mathbf{F}_{i}$ of these forces. Note that, in this sum, the contributions from rigid bodies within the selected molecule cancel out.

- Rigid Body. When only one rigid body $\mathcal{R}_{i}$ from a molecule is selected, we compute the molecular force $\mathbf{F}_{i}$ applied to the rigid body by the environment as before, but add to this force the kinematic constraint forces, i.e. the forces which maintain the kinematic constraints between the selected rigid body and its topological neighbors [11].

This flexibility allows us to propose two control schemes to handle the coupling between the simulation and the haptic device, that we detail in the next section.

\section{BILATERAL COUPLING}

In order for our tool to be used by untrained users, the control schemes must fulfill several requirements. In particular, the manipulation of the molecule via the master arm must be intuitive. The forces felt by the user must reflect the interaction forces computed by the simulation. The coupling has therefore to be transparent, so that even small modifications on the force profile are rendered to the operator.

Regarding the specificities of the software described in the previous section, different bilateral couplings can be investigated, based indistinctly on impedance or admittance control.

\section{A. Direct Force Feedback control}

The Direct Force Feedback control scheme (DFF) appears as the most intuitive when the simulation's input is the position of the rigid body being manipulated and forces sent to the user are the ones computed by the simulation. It is represented in figure 3, using the continuous time Laplace variable $s$. 


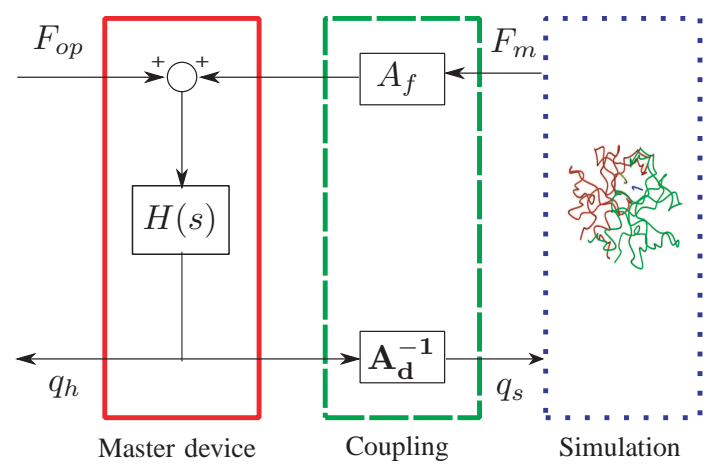

Fig. 3. DFF control scheme

The different parameters are:

- $\mathbf{A}_{\mathbf{d}}=\left[\begin{array}{cc}A_{d, T} & 0 \\ 0 & A_{d, R}\end{array}\right]$ where $A_{d, T}$ and $A_{d, R}$ are respectively used to scale down the positions and the rotations of the haptic handle. $A_{f}$ is used to enhance the forces sent to the user. These coefficients are necessary to adjust the scale difference between macro- and nanoworlds.

- $q_{s}=\left[x_{s}, \theta_{s}\right], q_{h}=\left[x_{h}, \theta_{h}\right]$ are respectively the position vectors of the rigid body's center of mass and of the haptic handle.

- $H(s)$ is the haptic device transfer function. It is composed of a mass and viscous friction [12].

The operator applies a force $F_{o p}$ on the master device. The position of the haptic device handle is scaled by $\mathbf{A}_{\mathbf{d}}$, and used as the desired position of the rigid body's center of mass. The orientation of the rigid body is computed with respect to the rotations of the haptic device handle. For a given time step, the user moves the rigid body to a desired position $q_{s}$. The simulator modifies this position using a criterion based on energy minimization and taking into account the influence of other atoms. This new position will be used as the reference position for the next time step. Thanks to this method, all the displacements make sense and represent the molecular dynamics.

If the user wants to manipulate multiple rigid bodies as an unique entity (for example to manipulate a flexible molecule at its center of mass), the same displacement and orientation are applied to all the rigid bodies. In that case, the simulation also updates the configuration of the rigid bodies based on internal and environmental interactions, and determines the new sub-tree of rigid bodies as described in section III.

The simulation remains stable if the displacements are small enough for a given time step, so that no atoms interpenetrate each other. The matrix $\mathbf{A}_{\mathbf{d}}$ is used to adjust the displacements of the user so that the simulation can deal with them. The molecular forces $F_{m}$ are sent to the user after being increased by a factor $A_{f}$.

\section{B. Force-Force control}

The second approach, Force-Force control $(F F)$, consists in using only forces for both manipulation of the rigid body and haptic feedback. This bilateral coupling is represented on figure 4.

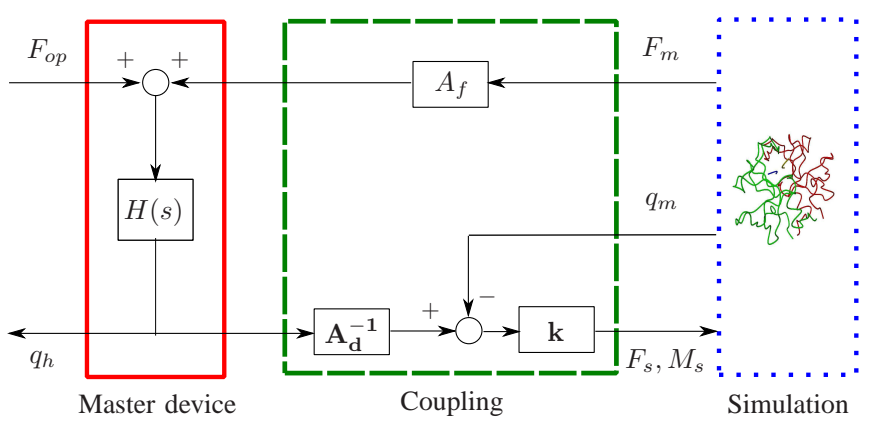

Fig. 4. FF control scheme

The parameter $\mathbf{k}=\left[\begin{array}{cc}k_{T} & 0 \\ 0 & k_{R}\end{array}\right]$ is a stiffness matrix used to tune the forces and the moments applied on the rigid body.

This control scheme is similar to the $D F F$ as the feedback path is also composed of molecular forces $F_{m}$ amplified by $A_{f}$.

The difference is that the user applies a force $F_{s}$ and a moment $M_{s}$ on the rigid body to manipulate it. These values are computed based on the position of the rigid body's center of mass $q_{m}$ and the position of the haptic handle $q_{h}$ :

$$
\begin{aligned}
& F_{s}=k_{T}\left(x_{h} / A_{d, T}-x_{m}\right) \\
& M_{s}=k_{R}\left(\theta_{h} / A_{d, R}-\theta_{m}\right)
\end{aligned}
$$

During a simulation step, the software takes into account the forces applied by the user on the rigid body, as well as the forces due to the interactions between atoms. These forces are used to derive the new position/orientation of the rigid body. Compared to the $D F F$, both the forces from the user and from the other atoms are considered simultaneously. The position does not need to be corrected afterwards, contrary to section III-A, and therefore is less subject to oscillations. When the user manipulates a molecule composed of several rigid bodies, the force applied on each of them is computed so that their accelerations are the same. This is done using adapted values of $\mathbf{k}$, and considering the mass of each rigid body. As previously, for flexible molecules, the configuration of these rigid bodies are updated at each time step using a criterion based on energy minimization.

Compared to the $D F F$ formulation, the ergonomy of the system is modified when a force is used as the simulator's input. The user does not control the position but the dynamics of the molecule. Depending on the application, users can choose between these two modes.

\section{SIMULATION RESULTS}

Experiments are performed on a $3 G H z$ processor Intel PC, using Windows operating system. The available haptic device is a Phantom Omni haptic interface from Sensable ${ }^{1}$. This master arm is a 6 degrees of freedom (dof) movement and a 3 dof force feedback device. It is controlled using the OpenHaptics development toolkit.

\footnotetext{
${ }^{1}$ http://www.sensable.com
} 


\section{A. Manipulation of molecules}

The tool presented offers the possibility to the user to interact in real time during the conception process of new molecular structures. This functionality is of primary importance to design new materials and alloys with specific properties. A first experiment is performed using the HIV protease and its inhibitors (pdb code: $2 \mathrm{AZZ}$ ) to demonstrate that our tool is adapted to such kind of manipulations.

The system considered is composed of 848 degrees of freedom. To simulate it in real time so that haptic feedback can be used, the adaptive feature of the algorithm is used, and only 432 dof are activated. This allows to simulate complex systems in a few dozens of milliseconds.

The manipulation performed consists in extracting an inhibitor of the molecule, and then reinserting it (see figure 5). It is repeated twice, using both of the bilateral couplings presented in section III.
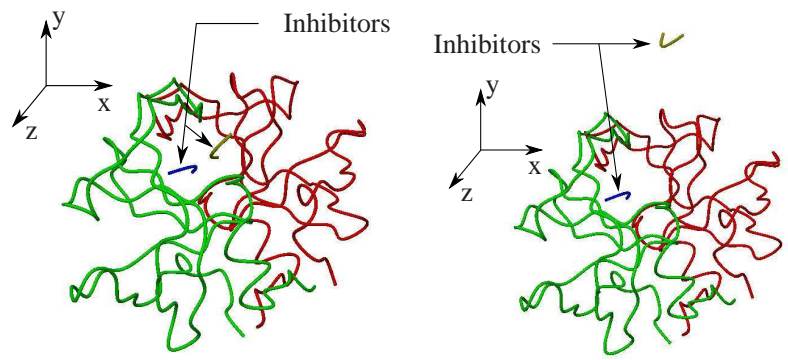

(a) Initial and final positions: in- (b) Intermediate position: one of the hibitors inserted in the HIV protease inhibitor is removed from the HIV protease

Fig. 5. Manipulation of one of the HIV protease's inhibitors using the haptic device

The molecule is manipulated as a whole, so that it would be equivalent for the user to manipulate its center of mass in the case of a rigid molecule. However, the molecule is indeed flexible, and can be deformed by the simulation (interactions with other molecules). Using this manipulation mode, even an untrained user can move precisely the HIV's inhibitor.

The movement performed by the user is mainly directed along the $y$ axis (see figure 5), so that it can easily be reproduced in order to compare the two control schemes. However, rotations are allowed.

The forces along the $x, y$ and $z$ axes are depicted in figures 6,7 and 8 . They are represented with respect to the position along the $y$ axis (direction of the main displacement).

The parameters of the controllers are chosen so that the forces delivered by the simulator (in the order of dozens of nanonewtons) are amplified to be easily felt by the user. To select the position scaling factors, a trade off between stability of the system and ease of manipulation due to the limited workspace of the haptic device must be considered. These issues have been partially addressed in our previous work [13].

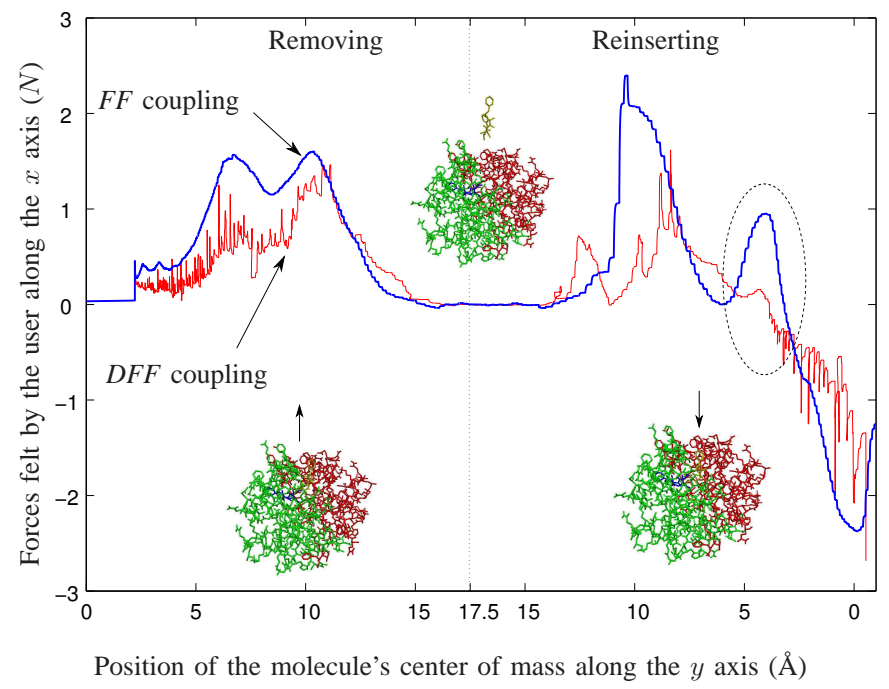

Fig. 6. Force felt by the user along the $x$ axis of the haptic interface while manipulating an inhibitor of the HIV molecule (interaction forces between molecules)

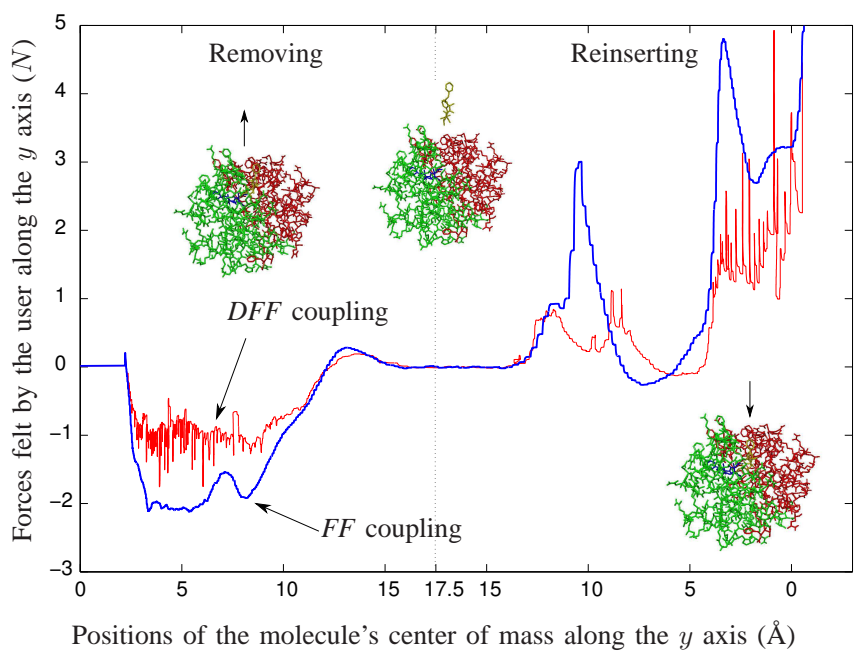

Fig. 7. Force felt by the user along the $y$ axis of the haptic interface while manipulating an inhibitor of the HIV molecule (interaction forces between molecules)

In this experiment, the force scaling factor is the same for both the $D F F$ and $F F$ control schemes:

$$
A_{f}=0.3 \cdot 10^{9}
$$

Regarding the velocity scaling factors, they are set to:

$\mathbf{A}_{\mathbf{d}}^{\mathbf{D F F}}=\left[\begin{array}{cc}0.25 \cdot 10^{9} & 0 \\ 0 & 5\end{array}\right] ; \mathbf{A}_{\mathbf{d}}^{\mathbf{F F}}=\left[\begin{array}{cc}0.025 \cdot 10^{9} & 0 \\ 0 & 5\end{array}\right]$

Forces obtained with the $D F F$ and $F F$ couplings are similar. Three phases can be clearly distinguished (corresponding to the three states of the molecule depicted in figures 6,7 and 8). The first phase is the extraction of one of the inhibitor, when it is situated at less than $15 \AA$ of its initial position. The second phase consists in removing the inhibitor from the rest of the structure. When the inhibitor is further than 15 $\AA$, no force is applied on it. The reinsertion into the system occurs when the position is again less than $15 \AA$. 


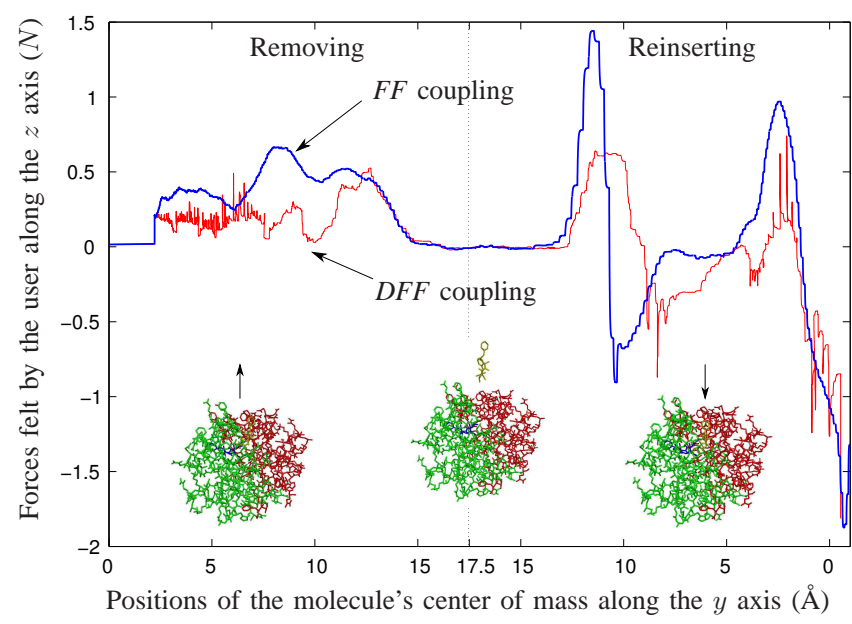

Fig. 8. Force felt by the user along the $z$ axis of the haptic interface while manipulating an inhibitor of the HIV molecule (interaction forces between molecules)

For both of the couplings, the magnitude of the forces is in the order of the newton so that the interaction forces can clearly be felt by the user. When the $D F F$ control scheme is used, some oscillations can be felt. As seen in section III, this is due to the structure of the simulation since the rigid body first takes the position set by the user. This position is then modified according to the interactions with other atoms. Adding damping and changing the structure of the coupling has been considered in previous work, but decreases the transparency [13].

Even if the forces felt using the $D F F$ and $F F$ are similar (especially on figures 7 and 8), some differences can be noted (e.g. dotted circle in figure 6). As stated earlier, rotations are free. For a given position along the $y$ axis, the orientation can be different. The interactions between the manipulated inhibitor and the HIV protease are thus not the same which explains the differences on the force fed back.

As demonstrated in this example, using both of the couplings, the system remains stable and the forces felt by the user allow him or her to clearly distinguish the interactions between the molecules. Compared to [5], no damping is added in our system which allows a good transparency. Even untrained users did not experience any difficulty in using either position or force control mode. Therefore, our system can indeed be used during a conception process of molecular structures, or to test docking properties of a ligand.

\section{B. Internal forces}

The tool that we present in this paper is not limited to molecular interactions. It can also be useful to manipulate a single rigid body of a flexible molecule, and feeling the internal forces and the inertial effects. This is useful in particular to synthesize a new molecule by increasing progressively the number of atoms. Therefore, it is possible to change the topology of the molecule and to control its specificities.

The experiment presented in this paragraph consists in manipulating a single rigid body of a flexible molecule, and feeling internal forces and inertia. No other molecules are present. To perform this experiment, the user selects a rigid body of an inhibitor of the HIV virus. Using the haptic device and the $F F$ coupling, he or she applies a force on this inhibitor to unfold it. The other extremity is fixed. When the molecule is stretched, the user moves back the haptic device to the initial position. Doing so, the molecule is refolded. Since the molecule is composed of only 17 degrees of freedom, the adaptive feature of the algorithm is not useful to obtain a fast simulation of the whole molecule (the simulation's sampling period is $14 \mathrm{~ms}$ with all the dof activated). The same position scaling factor $\mathbf{A}_{\mathbf{d}}^{\mathbf{F F}}$ than in section IV-A is used. The force scaling factor is $A_{f}=0.1 \cdot 10^{9}$.

The figure 9 represents the norm of the force felt by the user against the norm of the position of the center of mass.

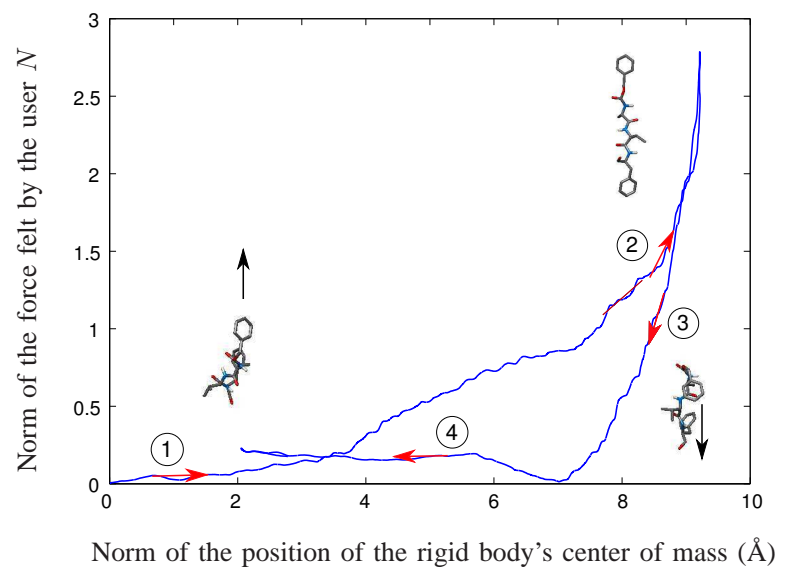

Fig. 9. Force felt by the user while unfolding an inhibitor of the HIV virus (internal forces). Non adaptive simulation

First, the force increases as the molecule is stretched (part 1). When the molecule is deployed, the user applies an additional force and the internal forces increase (part 2 ). Then, the user moves back the rigid body and he or she releases the constraint applied on it. The forces quickly decrease because of the reduction of internal forces (part 3). Finally, the molecule is moved toward its initial position. The interaction forces due to the other rigid bodies are felt (part 4).

These results show that our system is able to render small variations of the forces to the user (in the order of dozens of nanonewtons). It demonstrates that the system is well suited to achieve high transparency. The new possibility to manipulate a single rigid body of a molecule has been asked by Sanofi-Aventis. With this possibility, one can expect to evaluate in real-time the influence of the molecular structure on its dynamics.

\section{CONCLUSION}

We have presented a new tool to perform molecular simulations with haptic feedback. Using the force feedback 
device, the user can manipulate the entire HIV inhibitor. The simulator allows for real-time computation of the forces thanks to an adaptive method. To provide flexibility, two different manipulation modes are proposed (position and force) thanks to two different bilateral couplings. Both of them allow the operator to feel interaction forces.

All the stages of the design process of specific molecular structures can be handled by our new system. The internal forces of a molecule can be felt by the user. This possibility may be relevant for studying the possible conformational changes of a molecule and its properties. The experiment we performed, and which has been tested by Sanofi-Aventis engineers, shows that the simulator and the coupling achieve high performances. Indeed, the low values of the internal forces are felt by the user in a transparent and stable way.

This paper demonstrates the first results obtained with this system. User-based tests must be carried out to evaluate the effectiveness of the proposed system compared to conventional devices such as a mouse or a joystick. Tests should also be carried out to compare complex manipulation tasks performed with or without haptic feedback.

\section{ACKNOWLEDGMENT}

This work was supported by the French National Agency of Research, through the PACMAN project.

\section{REFERENCES}

[1] M. Ouh-young, M. Pique, J. Hughes, N. Srinivasan, and F. P. Brooks, "Using a manipulator for force display in molecular docking," in Proceedings of the IEEE International Conference on Robotics and Automation, April 1988, pp. 1824-1829.

[2] B. Daunay, A. Micaelli, and S. Régnier, "Energy-field reconstruction for haptic-based molecular docking using energy minimization processes," in Proceedings of the IEEE International Conference on Intelligent Robots and Systems, October 2007, pp. 2704-2709.

[3] S. K. Lai-Yuen and Y.-S. Lee, "Energy-field optimization and hapticbased molecular docking and assembly search system for computeraided molecular design (camd)," in Proceedings of the IEEE Symposium on Haptic Interfaces for Virtual Environment and Teleoperator Systems, March 2006, pp. 233-240.

[4] M. Teodoro, G. Phillips, and L. Kavraki, "Molecular docking: a problem with thousands of degrees of freedom," in Proceedings of the IEEE International Conference on Robotics and Automation, May 2001, pp. 960-965.

[5] B. Daunay, A. Micaelli, and S. Régnier, "6 dof haptic feedback for molecular docking using wave variables," in Proceedings of the IEEE International Conference on Robotics and Automation, April 2007, pp. $840-845$.

[6] M. Moore, A. Enomoto, T. Nakano, Y. Okaie, and T. Suda, "Interfacing with nanomachines through molecular communication," IEEE International Conference on Systems, Man and Cybernetics, pp. 1823, October 2007.

[7] B. Atakan and O. Akan, "An information theoretical approach for molecular communication," Bio-Inspired Models of Network, Information and Computing Systems, pp. 33-40, December 2007.

[8] D. D. Rossi, A. Ahluwalia, and M. Mule, "Molecular and supramolecular systems for sensing and actuation," IEEE Engineering in Medicine and Biology Magazine, vol. 13, no. 1, pp. 103-111, February 1994.

[9] T. Grove, K. Puckett, N. Brunet, G. Mihajlovic, L. McFadden, X. Peng, S. von Molnar, T. Moerland, and P. Chase, "Packaging actomyosinbased biomolecular motor-driven devices for nanoactuator applications," IEEE Transactions on Advanced Packaging, vol. 28, no. 4, pp. 556-563, November 2005.

[10] S. Morin and S. Redon, "A force-feedback algorithm for adaptive articulated-body dynamics simulation," in Proceedings of the IEEE International Conference on Robotics and Automation, April 2007, pp. 3245-3250.
[11] R. Rossi, M. Isorce, S. Morin, J. Flocard, K. Arumugam, S. Crouzy, M. Vivaudou, and S. Redon, "Adaptive torsion-angle quasi-statics: a general simulation method with applications to protein structure analysis and design," in International Conference on Intelligent Systems for Molecular Biology, vol. 23, July 2007, pp. i408-i417.

[12] N. Diolaiti, G. Niemeyer, F. Barbagli, and J. Salisbury, "Stability of haptic rendering: Discretization, quantization, time delay, and coulomb effects," IEEE Transactions on Robotics, vol. 22, no. 2, pp. 256-268, April 2006.

[13] A. Bolopion, B. Cagneau, D. S. Haliyo, and S. Régnier, "Tuning the gains of haptic couplings to improve force feedback stability in nanorobotics," in Proceedings of the IEEE International Conference on Robotics and Automation, May 2009, pp. 4320-4325. 